Research Paper

\title{
MicroRNA Signature of Lung Adenocarcinoma with EGFR Exon 19 Deletion
}

\author{
Lixia Ju${ }^{1 凶}$, Mingquan Han¹, Xuefei $\mathrm{Li}^{2}$, Chao Zhao ${ }^{2}$ \\ 1. Department of Integrative Medicine, Shanghai Pulmonary Hospital, Tongji University School of Medicine, Tongji University, Shanghai, People's Republic of \\ China; \\ 2. Department of Lung Cancer and Immunology, Shanghai Pulmonary Hospital, Tongji University Medical School Cancer Institute, Tongji University, \\ Shanghai, People's Republic of China.
}

$\square$ Corresponding author: Lixia Ju, MD, PhD, Department of Integrative Medicine, Shanghai Pulmonary Hospital, Tongji University School of Medicine, No. 507 Zheng Min Road, Shanghai 200433, People's Republic of China. E-mail: jvlixia@126.com.

(1) Ivyspring International Publisher. This is an open access article distributed under the terms of the Creative Commons Attribution (CC BY-NC) license (https://creativecommons.org/licenses/by-nc/4.0/). See http://ivyspring.com/terms for full terms and conditions.

Received: 2016.10.04; Accepted: 2017.03.06; Published: 2017.05.11

\begin{abstract}
The findings of EGFR mutations and the development of targeted therapies have significantly improved the overall survival of lung cancer patients. Still, the prognosis remains poor, so we need to know more about the genetic alterations in lung cancer. MicroRNAs are dysregulated in lung cancer, and some of them can regulate EGFR. So it is very important to predict the candidate microRNAs that target mutated EGFR and to investigate the role of these candidate microRNAs in lung cancer. In this study, we investigated the difference of microRNAs expression between lung adenocarcinoma cell lines with EGFR exon 19 deletion ( $\mathrm{H} 1650$ and $\mathrm{PC} 9)$ and wild-type $(\mathrm{H} 1299$ and A549) using the Phalanx Human Whole Genome Microarray. Then the expression of individual microRNAs was validated by qRT-PCR assays. Moreover, we detected the microRNAs expression in plasma of lung adenocarcinoma patients with EGFR exon 19 deletion and wild-type. Lastly, we explored the function of the positive microRNA in EGFR tyrosine kinase inhibitors (EGFR-TKIs) resistance using MTT and Annexin V-APC assays. The expression of 1,732 microRNAs was evaluated, and we found that microRNAs expression was different between these two groups. Hsa-miR-141-3p, hsa-miR-200c-3p, hsa-miR-203, hsa-miR-3182, hsa-miR-934 were up-regulated and hsa-miR-3196 was down-regulated in the EGFR exon 19 deletion group compared with wild-type group. The detection of circulating microRNAs showed that miR-3196 was down-regulated in lung adenocarcinoma patients with EGFR exon 19 deletion compared with wild-type. And then the MTT assay results showed that miR-3196 had no effect on the sensitivity of erlotinib. The results of apoptosis analysis showed that inhibition of miR-3196 and erlotinib induced more apoptosis in H1299 cells than erlotinib alone, and overexpressed miR-3196 and erlotinib induced less apoptosis in PC9 cells than erlotinib alone $(P<0.05)$. It is suggested that microRNAs associate with EGFR exon 19 deletion and miR-3196 may be further explored as a potential predictor and targeted biomarker when it is difficult to get the tumors.
\end{abstract}

Key words: Genome, microRNA, EGFR, exon 19 deletion, Adenocarcinoma.

\section{Introduction}

Lung cancer, one of the most common malignant tumors [1], leads to the most cancer death in the world. The prevalence and mortality of the lung cancer are still rising. In all new diagnosed lung cancer, non-small cell lung cancer (NSCLC) accounts for approximately 85\%, which varies both in molecular and clinical presentation. In spite of years of research, the 5 -year survival is only about $18 \%$ [2]. The discovery of key driver mutations has led to a more personalized approach in the treatment of advanced lung cancer. The sensitive mutations of EGFR indicate potential sensitivity to EGFR-TKIs. EGFR exon 19 deletion and L858R substitution in exon 21 have been extensively proved to be the sensitive 
mutations. However, the efficacy of EGFR-TKIs varies among different sensitive EGFR mutations. Several studies have reported that the advanced NSCLC patients with EGFR exon 19 deletion had a longer overall survival (OS) and/or progression-free survival (PFS) with the treatment of gefitinib or erlotinib compared with those with the L858R mutation [3-6].

MicroRNAs are evolutionarily conserved, endogenous small non-coding RNAs with 18-25 nucleotides that have important functions in diverse biological processes, such as cell proliferation, differentiation, and apoptosis [7-9]. Furthermore, microRNAs play an essential role in behaving as either oncogenes or tumor suppressor genes [10]. Increasing evidence indicates that dysregulation of specific microRNAs contributes to the development and progression of cancer, including lung cancer [11-13]. Moreover, microRNAs can regulate EGFR [14]. So it is important to identify the candidate microRNAs regulating EGFR exon 19 deletion because it might spark the design of novel therapeutics to combat the resistance to EGFR-TKIs or develop new targeted therapy.

In this study, we conducted an explorative microRNAs expression study with two groups of lung adenocarcinoma cell lines, including EGFR exon 19 deletion group (H1650 and PC9) and EGFR wild-type group (A549 and H1299), using microRNA microarrays. Our main focus was the different microRNAs in the two groups. The selected microRNAs were confirmed by qRT-PCR in the cell lines, and then we measured 3 microRNAs expressed differently in cell lines in the plasma of 14 non-smoking female lung adenocarcinoma patients with wild-type EGFR and 13 other patients with EGFR 19 deletion. Accordingly we discussed the association of microRNAs with EGFR exon 19 deletion.

\section{Patients and Methods}

\section{Cell lines and cell culture}

Human adenocarcinoma cell lines PC9 and H1650 (harboring EGFR exon 19 deletion) and A549 and H1299 (harboring wild-type EGFR) were provided by Cancer Institute of Tongji University Medical School, China. All these cells were cultured at $37^{\circ} \mathrm{C}$ with $5 \% \mathrm{CO}_{2}$ in Dulbecco's modified Eagle's medium (DMEM) supplemented with 10\% fetal bovine plasma (FBS), $100 \mathrm{U} / \mathrm{ml}$ penicillin and 100 $\mathrm{mg} / \mathrm{ml}$ streptomycin.

\section{Patient enrollment and plasma samples}

All patients in the study were recruited from Shanghai Pulmonary Hospital, Tongji University
Medical School, between January 2012 and June 2014. The patients were newly diagnosed and histologically confirmed primary lung adenocarcinoma. Patients with the previous history of cancer, radiotherapy or chemotherapy were excluded. The study was approved by the ethical review committee at Tongji University Institutional Care and Use Committee. All blood plasma samples were collected and put into a liquid nitrogen tank for long-term storage until microRNAs extraction.

\section{Total RNA isolation}

Total RNA was extracted from cells using TRIZOL Reagent (Invitrogen, USA). The RNA concentration and purity were accessed by OD260/OD280 ( $\geq 1.6)$ and OD260/OD230 ( $\geq 1.0)$, and the RNA yield and quality were checked ( $R I N \geq 5.0$ ) using Agilent 2100 Bioanalyzer (Agilent Technologies, Santa Clara, CA, USA).

\section{Human microRNAs OneArray®}

Human microRNA OneArray ${ }^{\circledR}$ v3 (Phalanx Biotech Group, Taiwan) contains triplicated 1,711 unique microRNA probes from Human (miRBase Release v17), each printed in technical triplicate, and 189 experimental control probes.

\section{Microarray analysis}

Small RNA was pre-enriched by Nanoseplook (Pall Corporation, USA) from $2.5 \mu \mathrm{g}$ total RNA samples and labeled with microRNAs ULSTM Labeling Kit (Kreatech Diagnostics, The Netherlands). Labeled targets were hybridized to the Human microRNA OneArray ${ }^{\circledR} \quad$ v3 with OneArray ${ }^{\circledR}$ Hybridization System. After 16 hours hybridization at $37^{\circ} \mathrm{C}$, non-specific binding targets were washed away by three different washing steps (Wash $37{ }^{\circ} \mathrm{C} 5$ mins; WashII $37{ }^{\circ} \mathrm{C}, 5$ mins $25{ }^{\circ} \mathrm{C} 5$ mins; Wash III rinse 20 times), and the slides were dried by centrifugation and scanned by an Axon 4000B scanner (Molecular Devices, Sunnyvale, CA, USA). Normalized spot intensities were transformed to gene expression log2 ratios between the mutation and wild-type group. The spots with $\log 2$ ratio $\geq 1$ or $\log 2$ ratio $\leq-1$ and $P$-value $<0.05$ were tested for further analysis.

\section{Validation by qRT-PCR}

Quantitative real time-PCR (qRT-PCR) was carried out on the 7900HT thermocycler (Applied Biosystems, Foster City, CA). U6 was used for internal controls. The data were managed using the Applied Biosystems software RQ. The relative levels of the microRNAs in cells were normalized to U6. By using the 2- $\triangle \Delta \mathrm{CT}$ equation, microRNA amount was calculated relative to the internal control U6. 


\section{EGFR mutation analyses}

Mutation analyses of EGFR exons 18-21 were performed on 27 of the tumor samples using the amplification-refractory mutation system assay (ARMS). Data analyses were performed by employing the LightCycler Adapt software (LightCycler 480 Software, v. 1.5).

\section{Digital PCR}

Digital PCR was performed in parallel for the measurement of microRNAs in the serially-diluted oligonucleotides. $30 \mu \mathrm{L}$ of the reaction mixture containing $15 \mu \mathrm{L}$ QuantStudio ${ }^{\mathrm{TM}}$ 3D Digital PCR Master Mix, 2X (Life technologies), $2 \mu \mathrm{L}$ of cDNA solution, and $1.5 \mu \mathrm{L}$ of TaqManR Assay, 20X (primer/probe mix) (Life technologies) and $11.5 \mu \mathrm{L}$ water. The droplets generated from each sample were transferred to a 96-well PCR plate (Eppendorf, Germany). PCR amplification was carried on a T100 thermal cycler (QuantStudio ${ }^{\mathrm{TM}}$ 3D Digital PCR System) at $96^{\circ} \mathrm{Cfor} 10 \mathrm{~min}$, followed by 39 cycles $60^{\circ} \mathrm{C}$ for $2 \mathrm{~min}$, then $98^{\circ} \mathrm{C}$ for $0.5 \mathrm{~min}$. For Final extension, followed by $60^{\circ} \mathrm{C}$ for $2 \mathrm{~min}$, Last, Storage is at $10^{\circ} \mathrm{C}$ for 100 min. $^{15}$

\section{Lentivirus production and transduction}

The hsa-miR-3196 plasmids and the controls were synthesized by GeneChem (Shanghai, China). The viruses were packaged in 293T cells according to standard protocols and the virus particles were harvested $72 \mathrm{~h}$ later. The packaged lentiviruses were named LV-hsa-miR-3196-inhibition (19097-1) and LV-hsa-miR-3196 (19155-1). The empty lentiviral vector CON053, CON238 were used as negative controls. A549, H1299 and PC9, H1650 cells were infected with virus particles plus $8 \mu \mathrm{g} / \mathrm{ml}$ Polybrene (Sigma, St. Louis, Missouri, USA). Infected cells were selected with puromycin for up to 7 days.

\section{MTT assay}

To examine the therapeutic effect of miR-3196 inhibition and restoration, H1299, A549 and PC9, H1650 cells were planted at a density of $4 \times 10^{3}$ in 96-well plates and administered with erlotinib or solvent for $72 \mathrm{~h}$. On the day of harvest, $100 \mu \mathrm{l}$ of spent medium was replaced with an equal volume of fresh medium containing MTT $0.5 \mathrm{mg} / \mathrm{ml}$. Plates were incubated at $37^{\circ} \mathrm{C}$ for $4 \mathrm{~h}$, and then the medium was replaced by $100 \mu \mathrm{l}$ of dimethyl sulfoxide (Sigma, St Louis, MO, USA) and plates shaken at room temperature for $10 \mathrm{~min}$. The absorbance was measured at $570 \mathrm{~nm}$.

\section{Annexin V-APC assays}

Flow cytometry were used for apoptosis assay.
In flow cytometry assay, cells were plated in six-well plates. Twenty-four hours later, erlotinib was added into experimental wells and incubated for another $72 \mathrm{~h}$. The cells were harvested, washed with PBS and resuspended in $500 \mu \mathrm{l}$ binding buffer. The Cell were stained with $5 \mu \mathrm{l}$ of Annexin V-APC and incubated for $5 \mathrm{~min}$ at room temperature in the dark. Quantification of apoptosis was determined by flow cytometry.

\section{Statistical analyses}

Statistical analyses were carried out using SPSS software version 17.0. Independent-sample t-test was used to compare means, $\mathrm{p}$ value $<0.05$ was considered statistically significant. All in vitro results were derived from at least three independent experiments.

\section{Results}

\section{Microarrays analyses of cell lines with EGFR exon 19 deletion compared with wild-type}

To identify microRNAs that were differentially expressed between cell lines with EGFR exon 19 deletion and wild-type, the expression profiles of microRNAs (1,711 microRNAs) were assessed using microRNA microarrays. A subset of genes was selected for clustering analysis. An intensity filter was used to select genes where the difference between the maximum and minimum intensity values exceeds 200 among all microarrays. For this clustering analysis, the number of genes was 260. The differentially expressed genes were identified based on the threshold of fold change and p-value (Figure 1). Standard selection criteria was established at $\log 2$ $\mid$ Fold change $\mid \geq 0.8$ and $P<0.05$. The microRNAs differentially expressed between EGFR exon 19 deletion and wild-type are shown in Table $1,(P<$ 0.05). In total, let-7d-3p, miR-1307-5p, miR-141-3p, miR-200c-3p, miR-203, miR-3182, miR-4510, miR-934 were up-regulated in EGFR exon 19 deletion group compared with wild-type group. On the contrary, miR-3196, miR-4450, miR-4649-5p were down-regulated.

\section{Validation of the microarrays results using quantitative reverse transcriptase polymerase chain reaction ( $q R T-P C R$ )}

The eleven microRNAs, differentially expressed in the two cell groups in microarray analyses, were validated by qRT-PCR. As a result, six microRNAs expressed differentially between the two cell groups were identified (see Table 2). Hsa-miR-141-3p, hsa-miR-200c-3p, hsa-miR-203, hsa-miR-3182, and hsa-miR-934 were up-regulated, while hsa-miR-3196 was down-regulated (Figure 2). 


\section{Pathway analyses}

Genes involved in the signaling pathways which were experimentally observed or highly predicted to be regulated by the selected microRNAs were searched for using the microRNA target filter in TargetScan Release 6.2. The six microRNAs that were differentially expressed between EGFR exon 19 deletion and wild-type cell lines were associated with 3181 mRNA targets. Two microRNAs (hsa-miR-200c-3p, hsa-miR-203) were experimentally confirmed to target EGFR and be associated with EGFR-TKIs resistance [16-17].

\section{Circulating microRNAs in relation to EGFR exon 19 deletion}

This study was conducted on 27 participants stratified into EGFR exon 19 deletion group and wild-type group. Selected candidates were non-smoking lung adenocarcinoma patients. It is well known that the sensitivity of qPCR for the detection of the low copy genes is not so high, as it only can resolve $\sim 1.5$-fold changes of nucleic acids [18]. Given that a portion of the cancer-related microRNAs is derived from primary tumor and could be 'diluted' in the normal microRNAs [19-21], the microRNAs presenting at low levels in plasma could be undetectable by qPCR.

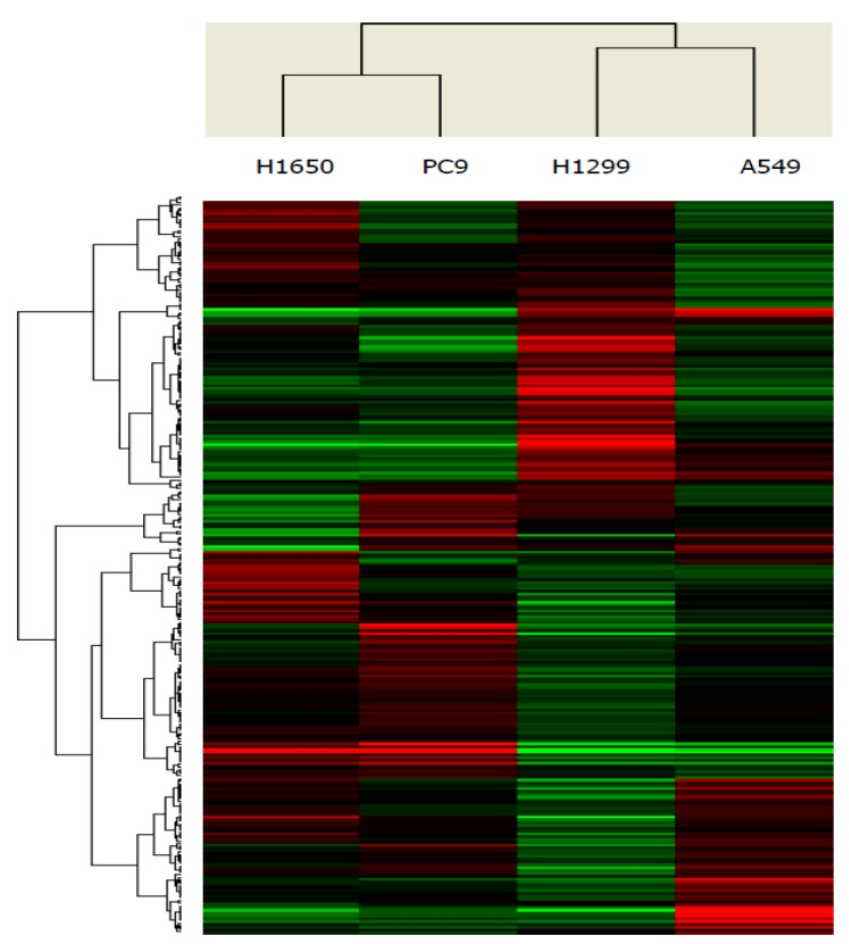

Figure 1. Unsupervised clustering of 260 microRNAs (rows) in 4 lung adenocarcinoma cell lines (columns). Up- and down-regulated genes are represented in red and green colors, respectively.

Table 1. MicroRNAs that were significantly differentially expressed between EGFR exon 19 deletion compared with wild-type cell lines

\begin{tabular}{llll}
\hline & MicroRNA & log2 (Ratio) Mutant/WT & P-value \\
\hline EGFR exon 19 deletion vs. wild-type lung adenocarcinoma cell lines (up-regulated) & & 0.040585 \\
1 & hsa-let-7d-3p & 0.647145 & 0.040504 \\
2 & hsa-miR-1307-5p & 0.927271 & 0.025595 \\
3 & hsa-miR-141-3p & 4.568754 & 0.00012 \\
4 & hsa-miR-200c-3p & 5.145011 & 0.01194 \\
5 & hsa-miR-203 & 1.932793 & 0.027437 \\
6 & hsa-miR-3182 & 0.92985 & 0.041041 \\
7 & hsa-miR-4510 & 1.097083 & 0.040343 \\
8 & hsa-miR-934 & 2.629921 & \\
EGFR exon 19 deletion vs. wild-type lung adenocarcinoma cell lines (down-regulated) & \\
1 & hsa-miR-3196 & & 0.02772 \\
2 & hsa-miR-4450 & -1.042712 & 0.016643 \\
3 & hsa-miR-4649-5p & -2.423635 & 0.047984 \\
\hline
\end{tabular}

Table 2. Validation of the microRNAs that were significantly differentially expressed between EGFR exon 19 deletion compared with wild-type cell lines (qRT-PCR)

\begin{tabular}{|c|c|c|c|c|c|}
\hline \multirow{2}{*}{\multicolumn{2}{|c|}{ MicroRNA }} & \multicolumn{4}{|c|}{$2^{-\Delta \Delta_{\mathrm{CT}}}$} \\
\hline & & A549 & H1299 & H1650 & PC9 \\
\hline \multicolumn{6}{|c|}{ EGFR exon 19 deletion vs. wild-type lung adenocarcinoma cell lines (up-regulated) } \\
\hline 1 & hsa-miR-141-3p & 1 & 0.501157 & 2.941734 & 8.224911 \\
\hline 2 & hsa-miR-200c-3p & 1 & 0.203063 & 405.4368 & 314.4456 \\
\hline 3 & hsa-miR-203 & 1 & 0.66742 & 9.426137 & 33.74652 \\
\hline 4 & hsa-miR-3182 & 1 & 0.326842 & 1.52979 & 0.532185 \\
\hline 5 & hsa-miR-934 & 1 & 0.429283 & 1.076738 & 1.815038 \\
\hline \multicolumn{6}{|c|}{ EGFR exon 19 deletion vs. wild-type lung adenocarcinoma cell lines (down-regulated) } \\
\hline 6 & hsa-miR-3196 & 1 & 0.33371 & 0.13742 & 0.314253 \\
\hline
\end{tabular}


A

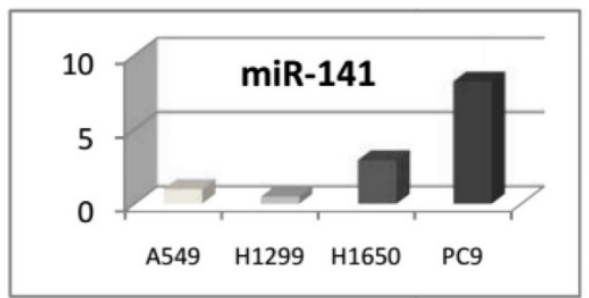

C

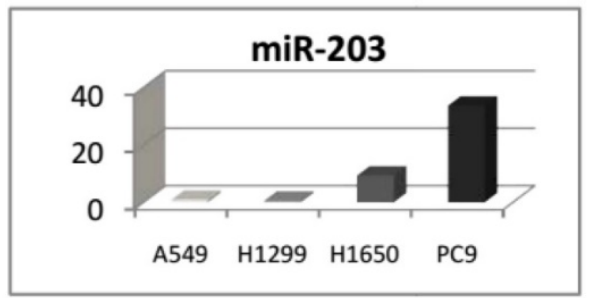

$\mathrm{E}$

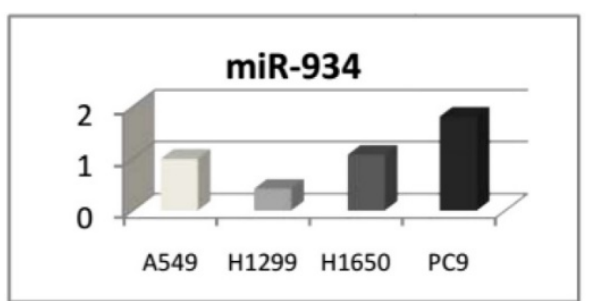

B

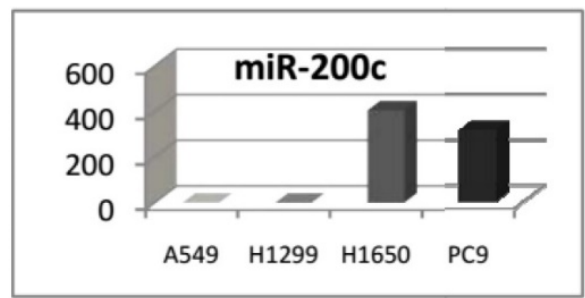

D

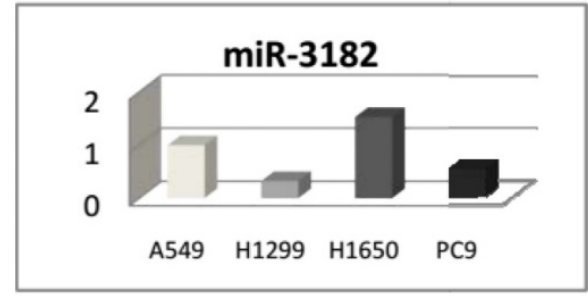

$\mathrm{F}$

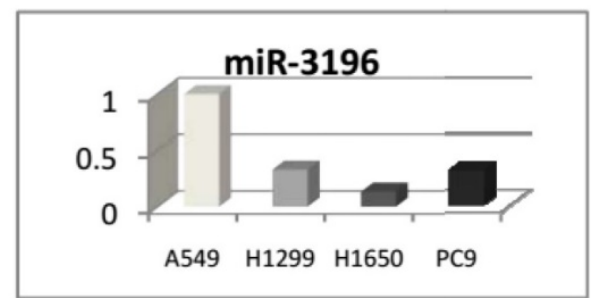

Figure 2. Six microRNAs were identified and differentially expressed between the two cell groups with EGFR exon 19 deletion and wild-type using qRT-PCR. A: Hsa-miR-141-3p, B: hsa-miR-200c-3p, C: hsa-miR-203, D: hsa-miR-3182, and E: hsa-miR-934 were up-regulated, and F: hsa-miR-3196 was down-regulated.

Circulating miR-3196 in relation to EGFR mutation status
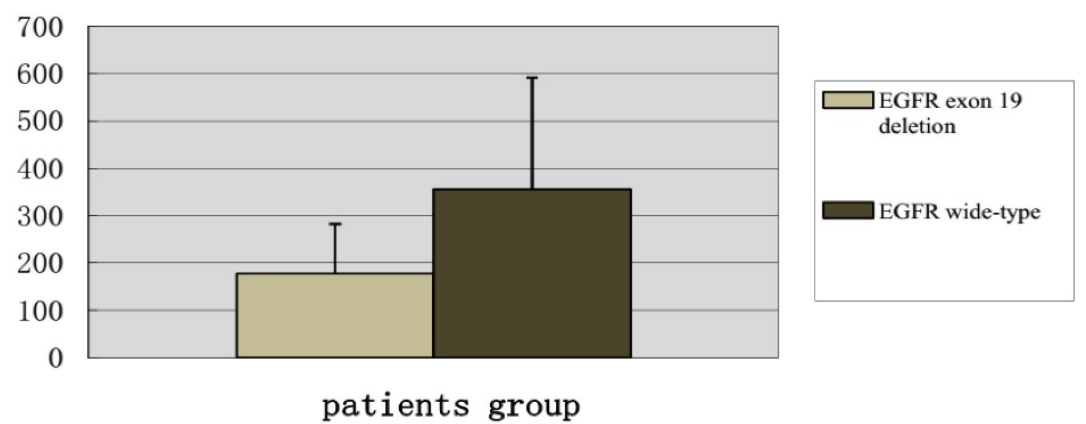

Figure 3. Digital PCR results showed that the circulating hsa-miR-3196 was down-regulated in plasma of lung adenocarcinoma patients with EGFR exon 19 deletion compared with wild-type.

Droplet digital PCR is a direct method for quantitatively detecting nucleic acids [22, 23]. It depends on limit proportion of the PCR volume, in which a positive microreactions indicates the presence of a single molecule in a given reaction. The number of positive reactions, together with Poisson's distribution, can be used to calculate the original target concentration in a straight and high-confidence measurement method [24].

In our study, digital PCR was used to detect three circulating microRNAs (hsa-miR-200c-3p, hsa-miR-203, and hsa-miR-3196) of 27 patients, with
13 patients with EGFR exon 19 deletion and 14 wild-type EGFR. In the results, we found that hsa-miR-3196 was down-regulated in the group of EGFR exon 19 deletion compared with wild-type, which was consistent with our results of cell study (Figure 3).

\section{Effect of miR-3196 on the sensitivity of erlotinib}

The miR-3196 was down-regulated in H1299 and A549 cell lines using LV-hsa-miR-3196-inhibition and was up-regulated in PC9 and H1650 cell lines using 
A

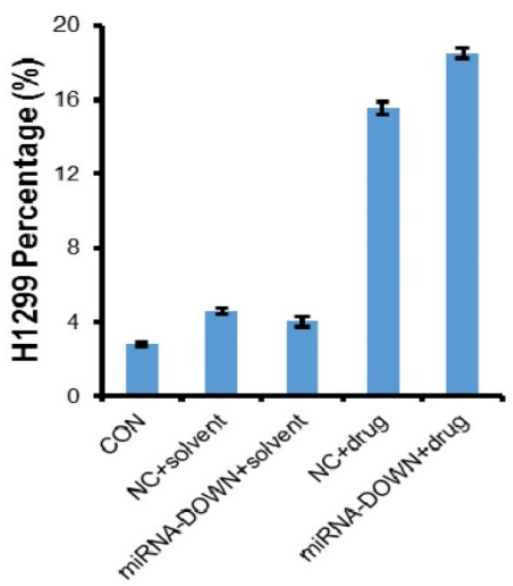

B

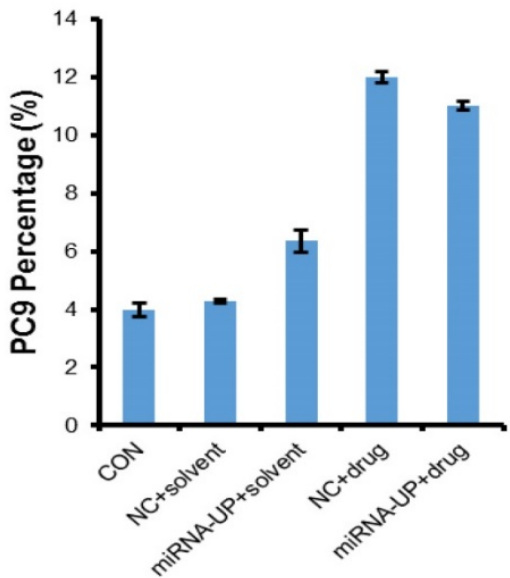

Figure 4. Apoptosis of $\mathrm{H1} 299$ and PC9 cell lines. The cells were treated with erlotinib for $72 \mathrm{~h}$ and then apoptosis was determined by flow cytometry. CON: control; NC: negative control; miRNA-DOWN: down-regulated miRNA group; miRNA-UP: up-regulated miRNA group. A: In H1299 cells, compared to the $\mathrm{NC}+$ solvent group, the number of apoptotic cells in $\mathrm{NC}+$ drug group was more $(\mathrm{P}<0.05)$; Compared to the miRNA-DOWN+solvent group, the number of apoptotic cells in miRNA-DOWN+drug group was more $(\mathrm{P}<0.05)$; Compared to the NC+ drug group, the number of apoptotic cells in the miRNA-DOWN+drug group was more $(\mathrm{P}<0.05)$. B: Compared to the $\mathrm{NC}+$ solvent group, the number of apoptotic cells in $\mathrm{NC}+\mathrm{drug}$ group was more $(\mathrm{P}<0.05)$; Compared to the miRNA-UP+solvent group, the number of apoptotic cells in miRNA-UP+drug group was more $(\mathrm{P}<0.05)$; Compared to the NC+ drug group, the number of apoptotic cells in the miRNA-UP+drug group was less $(P<0.05)$.

LV-hsa-miR-3196 respectively. The transfection efficiency was confirmed by qRT-PCR. The expression of miR-3196 was up-regulated to 1.83 times in PC9, and 1.38 times in H1650, and down-regulated to 0.59 times in H1299, 0.63 times in A549 (Figure S1). The MTT results showed that miR-3196 had no effect on the sensitivity of erlotinib (Figure S2). The results of flow cytometry showed that the apoptosis induced by erlotinib in A549 and H1650 was less than 5\%, indicating that there was no significant apoptosis in these cells, which was judged as a negative result, maybe because the selected concentration of erlotinib and the transfection efficiency were low (Figure S3). Administered with LV-hsa-miR-3196-inhibition and erlotinib induced more apoptosis in H1299 cells than erlotinib alone, and administered with LV-hsa-miR-3196 and erlotinib induced less apoptosis in PC9 cells than erlotinib alone $(\mathrm{P}<0.05)$ (Figure 4).

\section{Discussion}

In the present work, we selected two cell lines with EGFR exon 19 deletion (PC9 and H1650) and two cell lines with wild-type EGFR (H1299 with a lack expression of p53 protein and A549 with KRAS mutation). There are many cell lines with wild-type EGFR, but these two cell lines were verified highly resistant to EGFR-TKIs. we found eleven microRNAs differentially expressed between EGFR exon 19 deletion and wild-type lung adenocarcinoma cell lines in the microarray analyses. Then the qRT-PCR results identified that hsa-miR-141-3p, hsa-miR-200c-3p, hsa-miR-203, hsa-miR-3182, and hsa-miR-934 were up-regulated, and hsa-miR-3196 was down-regulated in EGFR exon 19 deletion group compared with wild-type group. Moreover, we found that the circulating miR-3196 was down-regulated in the lung adenocarcinoma patients with EGFR exon 19 deletion compared with wild-type.

Presently, not much is known about microRNAs expression in lung adenocarcinomas with EGFR exon 19 deletion versus wild-type. Using microRNA microarrays, Dacic et al. analyzed six lung adenocarcinomas and found that miR-155 was up-regulated only in EGFR/KRAS-negative group, miR-25 was up-regulated only in EGFR-positive group and miR-495 was up-regulated only in KRAS-positive adenocarcinoma. In opposite, let-7g was down-regulated in all three groups, with more significant downregulation in EGFR/KRAS-negative adenocarcinoma [25]. Seike et al. identified 12 microRNAs differentially expressed between 6 EGFR-mutated tumors and 22 wild-type tumors [26]. Zhang et al. found that miR-122 were differentially expressed between wild and mutant EGFR carriers $(\mathrm{P}=0.018)$ [27]. But all these have not accurately made the relation of microRNAs and EGFR exon 19 deletion clear.

In our results, Hsa-miR-141-3p and hsa-miR$200 c-3 p$ were up-regulated in exon 19 deletion versus wild-type. MiR-141-3p is a miR-200 family member, which consists of five microRNAs located in two different clusters (miRs-200b/a/429 and miRs-200c/141) on chromosome 1 and 12 in humans. Tejero $\mathrm{R}$ et al. reported that high miR-141 and miR-200c expression were associated with shorter OS in NSCLC patients with adenocarcinoma through 
MET and angiogenesis [28]. MiR-141 and miR-200c expression were significantly up-regulated in NSCLC tissues, and its overexpression accelerated NSCLC cell proliferation in vitro and tumor growth in vivo $[29,30]$. In line with our results, miR-203 overexpression resulted in increased sensitivity to erlotinib-induced apoptosis in nude mice after two weeks of treatment [31]. Siu MK's results demonstrated that the induction of bone metastasis and TKI resistance require miR-203 down regulation, activation of the EGFR pathway via altered expression of EGFR ligands (EREG and TGFA) and anti-apoptotic proteins (API5, BIRC2, and TRIAP1). Importantly, a sufficient reconstitution of invasiveness and resistance to TKIs treatment was observed in cells transfected with anti-miR-203 [17]. Li $\mathrm{j}$ et al. reported that up-regulated miR-200c could regain the sensitivity to gefitinib in the EGFR wild-type cell lines and miR-200c could regulate epithelial to mesenchymal transition through PI3K/AKT and MEK/ERK Pathways. In 66 NSCLC patients with wild-type EGFR, high levels of miR-200c expression was associated with higher disease control rate (DCR), longer progression-free survival (PFS) and longer overall survival (OS) compared with low miR-200c expression subgroup [16].

The function of hsa-miR-934 is unknown, but it is located in intron 4 of the vestigial-like 1 (VGLL1) gene. The miR-934 was the most strongly up-regulated microRNA in triple-negative IDCs (61.5-fold increase with respect to ER+ breast carcinomas) [32]. Although studies about hsa-miR-3196 are rare, it was found that miR-3196 was down-regulated in basal cell carcinoma compared with nonlesional skin [33], and was also down-regulated in PTC patients with non-(131) I-avid lung metastases versus (131)I-avid lung metastases [34]. Regarding hsa-miR-3182, there is still no published study.

A large number of microRNAs have been found to be stably expressed in human serum and plasma $[35,36]$. Circulating microRNAs or their expression profiles have been proposed to be useful biomarkers of the diagnosis and prognosis of cancer. Overexpressed plasma miR-21 was associated with the poor survival, lymph node metastasis and advanced stage of NSCLC [37].

As previously stated, very little is known about miR-3196, but the data from the next two previous published studies pointed to the possibility that EGFR exon 19 deletion could have a distinct molecular identity. Bjaanaes MM et.al examined microRNA expression in 154 surgically resected lung adenocarcinomas and 20 corresponding normal lung tissue samples using Agilent microarrays and found that 17 microRNAs were differentially expressed between EGFR-mutated and EGFR wild-type tumors [38]. Recently, Zhang et al. reported that circulating miR-195 and miR-122 may play important roles in predicting the overall survival as well as predicting EGFR mutation status in non-smoking female patients with lung adenocarcinoma. Measuring plasma levels of miR-195 and miR-122 may have especial values for EGFR mutant patients with lung adenocarcinoma [27]. In this regard, hsa-miR-200c-3p, hsa-miR-203, and hsa-miR-3196 were selected to be measured the plasma level in adenocarcinoma patients. The reason of our selection was the high expression level in the cell lines. Lastly, hsa-miR-3196 was significantly differentially expressed between EGFR exon 19 deletion and wild-type lung adenocarcinomas, which was consistent with our result of cell lines. In order to know the function of miR-3196 in erlotinib sensitivity, we down-regulated it in H1299 and A549 and up-regulated it in $\mathrm{H} 1650$ and PC9 cell lines respectively. The results showed that down-regulation of miR-3196 induced more apoptosis and up-regulation of miR-3196 reduced the apoptosis.

Therefore, the circulating microRNAs may potentially provide a noninvasive strategy for predicting response to EGFR TKIs when it's difficult to get the tumor tissues. Moreover, we identified that microRNAs regulate EGFR exon 19 deletion and which may represent a clinically useful modality to treat TKI resistance in NCSLC patients.

\section{Supplementary Material}

Supplementary figures.

http://www.jcancer.org/v08p1311s1.pdf

\section{Acknowledgements}

This work was supported by The National Natural Science Foundation of China (81207106) and Shanghai Municipal Health Bureau Fund (20124Y123); and the Program of Science and Technology Commission of Shanghai Municipality (14401932200). We also thank Prof. Rongzheng Ren for his revision of the draft.

\section{Competing Interests}

The authors have declared that no competing interest exists.

\section{References}

1. Ferlay J, Shin HR, Bray F, et al. Estimates of worldwide burden of cancer in 2008: GLOBOCAN 2008. Int J Cancer. 2010; 127:2893-917.

2. Siegel RL, Miller KD, Jemal A. Cancer statistics, 2016. CA Cancer J Clin. 2016; 66:7-30.

3. Jackman DM, Yeap BY, Sequist LV, et al. Exon 19 deletion mutations of epidermal growth factor receptor are associated with prolonged survival in 
non-small cell lung cancer patients treated with erlotinib or erlotinib. Clin Cancer Res. 2006; 12:3908-14.

4. Riely GJ, Pao W, Pham D, et al. Clinical course of patients with non-small cell lung cancer and epidermal growth factor receptor exon 19 and exon 21 mutations treated with erlotinib or erlotinib. Clin Cancer Res. 2006; 12:839-44.

5. Goto K, Nishio M, Yamamoto N, et al. A prospective, phase II, open-label study (JO22903) of first-line erlotinib in Japanese patients with epidermal growth factor receptor (EGFR) mutation-positive advanced non-small-cell lung cancer (NSCLC). Lung Cancer. 2013; 82:109-14.

6. Zhang Y, Sheng J, Kang S, et al. Patients with exon 19 deletion were associated with longer progression-free survival compared to those with L858R mutation after first-line EGFR-TKIs for advanced non-small cell lung cancer: a meta-analysis. PLoS One. 2014; 9:e107161.

7. Ambros V. MicroRNA pathways in flies and worms: growth, death, fat, stress, and timing. Cell. 2003; 113:673-6.

8. Galasso M, Sana ME, Volinia S. Non-coding RNAs: a key to future personalized molecular therapy?. Genome Med. 2010; 2:12

9. Lu J, Getz G, Miska EA, et al. MicroRNA expression profiles classify human cancers. Nature. 2005; 435:834-8.

10. Srivastava SK, Bhardwaj A, Arora S, et al. MicroRNA-345 induces apoptosis in pancreatic cancer cells through potentiation of caspase-dependent and -independent pathways. Br J Cancer. 2015;113:660-8

11. Chen X, Ba Y, Ma L, et al. Characterization of microRNAs in plasma: a novel class of biomarkers for diagnosis of cancer and other diseases. Cell Res. 2008;18:997-1006.

12. Gao $\mathrm{W}$, Shen $\mathrm{H}$, Liu $\mathrm{L}$, et al. MiR-21 overexpression in human primary squamous cell lung carcinoma is associated with poor patient prognosis. J Cancer Res Clin Oncol. 2011;137:557-66.

13. Shen J, Todd NW, Zhang H, et al. Plasma microRNAs as potential biomarkers for non-small-cell lung cancer. Lab Invest. 2011; 91:579-87.

14. Wang F, Chan LW, Law HK, et al. Exploring microRNA-mediated alteration of EGFR signaling pathway in non-small cell lung cancer using an mRNA: miRNA regression model supported by target prediction databases. Genomics. 2014; 104:504-11.

15. Sun B, Tao L, Zheng YL. Simultaneous quantification of alternatively spliced transcripts in a single droplet digital PCR reaction. Biotechniques. 2014; 56:319-25.

16. $\mathrm{Li} \mathrm{J}, \mathrm{Li} \mathrm{X}$, Ren $\mathrm{S}$, et al. miR-200c overexpression is associated with better efficacy of EGFR-TKIs in non-small cell lung cancer patients with EGFR wild-type. Oncotarget. 2014; 5:7902-16.

17. Siu MK, Abou-Kheir W, Yin JJ, et al. Loss of EGFR signaling regulated miR-203 promotes prostate cancer bone metastasis and tyrosine kinase inhibitors resistance. Oncotarget. 2014; 5:3770-84

18. Guan $\mathrm{P}, \mathrm{Yin} \mathrm{Z}, \mathrm{Li} \mathrm{X}$, et al. Meta-analysis of human lung cancer microRNA expression profiling studies comparing cancer tissues with normal tissues. J Exp Clin Cancer Res. 2012;31:54.

19. Chen X, Ba Y, Ma L, et al. Characterization of microRNAs in plasma: a novel class of biomarkers for diagnosis of cancer and other diseases. Cell Res. 2008; 18:997-1006.

20. Mitchell PS, Parkin RK, Kroh EM, et al. Circulating microRNAs as stable blood-based markers for cancer detection. Proc Natl Acad Sci U S A. 2008;105:10513-8.

21. Pritchard CC, Kroh E, Wood B, et al. Blood cell origin of circulating microRNAs: a cautionary note for cancer biomarker studies. Cancer Prev Res (Phila). 2012;5:492-7.

22. Hayden RT, Gu Z, Ingersoll J, et al. Comparison of droplet digital PCR to real-time PCR for quantitative detection of cytomegalovirus. J Clin Microbiol. 2013;51:540-6.

23. Day E, Dear PH, McCaughan F. Digital PCR strategies in the development and analysis of molecular biomarkers for personalized medicine. Methods. 2013; 59:101-7.

24. Vogelstein B, Kinzler KW. Digital PCR. Proc Natl Acad Sci U S A. 1999; 96: 9236-41.

25. Dacic S, Kelly L, Shuai Y, et al. microRNA expression profiling of lung adenocarcinomas: correlation with mutational status. Mod Pathol. 2010; 23:1577-82.

26. Seike M, Goto A, Okano T, et al. MiR-21 is an EGFR-regulated anti-apoptotic factor in lung cancer in never-smokers. Proc Natl Acad Sci U S A. 2009; 106:12085-90.

27. Zhang H, Su Y, Xu F, et al. Circulating microRNAs in relation to EGFR status and survival of lung adenocarcinoma in female non-smokers. PLoS One. 2013;8:e81408.

28. Tejero R, Navarro A, Campayo M, et al. miR-141 and miR-200c as markers of overall survival in early stage non-small cell lung cancer adenocarcinoma. PLoS One. 2014; 9:e101899.

29. Mei Z, He Y, Feng J, et al. MicroRNA-141 promotes the proliferation of non-small cell lung cancer cells by regulating expression of PHLPP1 and PHLPP2. FEBS Lett. 2014; 588:3055-61.

30. Baffa R, Fassan M, Volinia S, et al. MicroRNA expression profiling of human metastatic cancers identifies cancer gene targets. J Pathol. 2009; 219:214-21.

31. Garofalo M, Romano G, Di Leva G, et al. EGFR and MET receptor tyrosine kinase-altered microRNA expression induces tumorigenesis and erlotinib resistance in lung cancers. Nat Med. 2011;18:74-82.
32. Castilla MÁ, López-García MÁ, Atienza MR et al VGLL1 expression is associated with a triple-negative basal-like phenotype in breast cancer. Endocr Relat Cancer. 2014; 21:587-99.

33. Sand M, Skrygan M, Sand D, et al. Expression of microRNAs in basal cell carcinoma. Br J Dermatol. 2012; 167:847-55.

34. Qiu ZL, Shen CT, Song HJ, et al. Differential expression profiling of circulation microRNAs in PTC patients with non-131I and 131I-avid lungs metastases: a pilot study. Nucl Med Biol. 2015; 42:499-504.

35. Chen H, Liu H, Zou H, et al. Evaluation of Plasma miR-21 and miR-152 as Diagnostic Biomarkers for Common Types of Human Cancers. J Cancer. 2016; 7:490-9.

36. Zhao Q, Deng S, Wang G, et al. A direct quantification method for measuring plasma MicroRNAs identified potential biomarkers for detecting metastatic breast cancer. Oncotarget. 2016;7:21865-74.

37. Liu XG, Zhu WY, Huang YY, et al. High expression of plasma miR-21 and tumor miR-200c associated with poor prognosis in patients with lung cancer. Med Oncol. 2012; 29:618-26.

38. Bjaanaes MM, Halvorsen AR, Solberg S, et al. Unique microRNA-profiles in EGFR-mutated lung adenocarcinomas. Int J Cancer. 2014; 135:1812-21. 